Check for updates

Cite this: RSC Adv., 2019, 9, 25836

\title{
Novel cationic tannin/glycosaminoglycan-based polyelectrolyte multilayers promote stem cells adhesion and proliferation $\uparrow$
}

\author{
Paulo C. F. da Câmara, ${ }^{\text {*ac }}$ Rosangela C. Balaban, ${ }^{a}$ Mohammadhasan Hedayati, (iD ${ }^{c}$ \\ Ketul C. Popat, (D) ${ }^{d}$ Alessandro F. Martins (D) ${ }^{* b c}$ and Matt J. Kipper (D) ${ }^{c}$
}

\begin{abstract}
Modifying materials with biocompatible surface coatings is an important method for controlling cell responses to biomaterials. In this work, tanfloc (TN), a cationic tannin-derivative polymer was assembled with heparin (HEP) and chondroitin sulfate (CS), using the layer-by-layer (LbL) approach, to build polyelectrolyte multilayers (PEMs) and to design cytocompatible coatings. LbL deposition was monitored through Fourier-transform surface plasmon resonance, and characterized by X-ray photoelectron spectroscopy, atomic force microscopy, and contact angle measurements. The response of human adipose-derived stem cells (ADSCS) was evaluated in vitro. All of the TN-containing PEMs exhibit cytocompatibility and support adhesion, proliferation, and the spreading of ADSCs after 7 days of culture. HEP-TN PEM assembly with 11 layers $\left(\mathrm{HEP}-\mathrm{TN}_{11}\right)$ supports the greatest rate of cell proliferation. When TN is the terminal layer of the PEM, the surfaces promote the spreading of ADSCs, indicating that the surface charge and PEM terminal layer are key determinants of the microenvironmental niche that control cellular response. The promotion of stem cell attachment and proliferation makes these surface coatings potentially useful for biomedical implants and regenerative medicine.
\end{abstract}

Received 23rd May 2019

Accepted 29th July 2019

DOI: $10.1039 / c 9 r a 03903 a$

rsc.li/rsc-advances candidate materials in tissue engineering and regenerative medicine. These properties include capacity to bind biochemical signals, tunable biodegradation, processability, and mechanical properties. Recently, we have proposed the use of condensed tannin polymers as potential biologically derived biomaterials, ${ }^{3}$ demonstrating that they can be processed with synthetic biodegradable polymers. Surface modification is an effective approach used to improve the cell responses to the surfaces of engineering materials and to develop surfaces with cytocompatibility, blood compatibility, and other biological activity. ${ }^{4-7}$ Two key challenges in tissue engineering are (1) developing microenvironmental niches that facilitate stem cell attachment, expansion, and self-renewal, and (2) translating these niches to the surfaces of biomaterials, using simple, scalable, and low-cost techniques. ${ }^{8}$ Surface chemistry and topography of biomaterials affect cell adhesion and proliferation.9,10 Therefore, surface properties such as wettability, roughness, surface charge, and chemical composition are crucial to proper cell function. These surface properties can be tuned to optimize cell responses leading to tissue regeneration. ${ }^{11}$

The layer-by-layer (LbL) method is a self-assembly technique used to build polyelectrolyte multilayers (PEMs). Polyelectrolyte multilayers have received attention in biomedical applications because they afford control over surface coatings at the nanoscale, and offer reproducibility, uniform deposition over any sized surface, ${ }^{12}$ control over surface chemistry, ${ }^{13}$ capacity to 
present biomolecules, ${ }^{14}$ and they can be applied on a wide variety of substrates such as glass, ${ }^{15}$ nano structures, ${ }^{4,16,17}$ metals, ${ }^{18}$ polymers, and composites (including bone). ${ }^{19,20}$ The LbL assembly of PEMs is accomplished by alternating the deposition of polycations and polyanions from solution, onto a charged surface. ${ }^{21}$ The selection of the polycation-polyanion pair affords a wide variety of surface chemistries to be introduced to many different types of substrate surfaces. By adjusting the deposition conditions, ${ }^{22,23}$ LbL assembly of PEMs has been demonstrated on many types of biomaterials to modulate cell responses, ${ }^{24}$ bacteria resistance, ${ }^{19,25-28}$ protein adhesion ${ }^{29,30}$ and other biological events. ${ }^{31,32}$

Glycosaminoglycans (GAGs) are polyanionic polysaccharides found in mammalian tissue attached to proteoglycans in the ECM. GAGs possess attractive characteristics for biomaterials such as non-toxicity, biodegradability, cytocompatibility, and capacity to enhance attachment and growth of mammalian cells. ${ }^{33}$ When used to modify biomaterials surfaces, GAGs also impart growth factor binding capacity, which can be used to stabilize and deliver important growth factors that regulate cell differentiation and wound healing activities. ${ }^{16,34-38}$ These features make GAGs excellent candidate materials to mimic the biochemical and biomechanical properties of the ECM. Heparin (HEP) and chondroitin sulfate (CS) are sulfated GAGs, which play an essential role for stabilizing growth factors by preventing enzymatic degradation and potentiating growth factor-receptor binding. ${ }^{39}$ GAGs have been combined with polycationic chitosan-based materials to modulate biomaterials properties. ${ }^{36,37,39-41}$ For example, Almodóvar et al. presented a new method to functionalize electrospun chitosan nanofibers by coating with heparin and $N, N, N$-trimethyl chitosan PEMs and growth factors (FGF-2) adsorbed on the PEM-coated nanofibers. ${ }^{17}$ The combination of surface nanotopography of the nanofibers with glycosaminoglycan-based PEMs enabled development of three-dimensional materials for stabilization of therapeutic growth factors that could be delivered in vitro and in vivo and enhanced cell response and tissue recovery. ${ }^{16,20,42}$ While chitosan is a biodegradable polysaccharide, compatible with mammalian cells, chitosan has poor solubility in aqueous solutions at neutral pH. Furthermore, chitosan is frequently derivatized using hazardous alkylating agents to improve its properties.

Condensed tannins are hydrophilic, plant-derived, polyphenols, known for their antibacterial and antioxidant properties. ${ }^{43}$ Tanfloc (TN) is a commercial amino-functionalized tannin-derivative, that behaves as a polycation in dilute acid aqueous solutions below $\mathrm{pH}$ 6.0. ${ }^{44} \mathrm{TN}$ is synthesized from condensed tannins in the presence of ammonium chloride and formic acid, following a Mannich-type reaction..$^{45} \mathrm{TN}$ is biodegradable, and has demonstrated minimal toxicity towards mammalian cells. ${ }^{3} \mathrm{TN}$ has only recently been proposed for use as a biomaterial. As such, there are no existing reports on GAGTN PEMs constructed by LBL destined for biomedical applications. Facchi et al. produced polyelectrolyte complexes (PECs) based on TN/alginate. ${ }^{46}$ These PECs showed cytocompatibility, induced proliferation of preosteoblastic mouse cells, and exhibited antimicrobial (against S. aureus and E. coli) and antioxidant activities which could minimize inflammatory responses of biomaterials. ${ }^{46}$ This work suggests that TN could be combined with other anionic polysaccharides, such as GAGs, to develop new TN-based materials in tissue engineering.

This work reports a new class of biomaterial surfaces to enhance biocompatibility and cell response, using HEP-TN and CS-TN PEMs. Since the surface properties of PEMs, such as hydrophilicity and surface charge can be dominated by the chemistry of the terminal layer, we prepared CS-terminated CSTN PEMs, and both HEP-terminated and TN-terminated HEPTN PEMs. For the first time, we report that TN-based PEMs can create microenvironments that support cell function. PEM formation was monitored by using Fourier-transform surface plasmon resonance in situ, X-ray photoelectron spectroscopy, atomic force microscopy, and contact angle measurements. The TN-GAG-rich PEMs are highly hydrophilic, exhibit cytocompatibility and support the adhesion and proliferation of human adipose-derived stem cells (ADSCs). We propose that TN may serve as a replacement polycation for chitosan in polysaccharide-based biomaterials. The capacity to promote cell attachment combining the PEM nanoscale structure allows the development of surface coatings for biomaterials that can enhance tissue healing for tissue engineering and regenerative medicine.

\section{Materials and methods}

The amino-functionalized tannin, commercially marketed as tanfloc (TN) was donated by Tanac SA Company (MontenegroRS, Brazil). TN has been synthetized from condensed tannins extracted from the black wattle in Brazil. ${ }^{46} \mathrm{TN}$ is a moderate-tohigh molecular weight polymer $\left(\sim 600000 \mathrm{~g} \mathrm{~mol}^{-1}\right) .{ }^{44}$ Sodium chondroitin sulfate (CS) (from shark cartilage, containing 6\% sulfur, 6-sulfate $/ 4$-sulfate ratio $=1.24, M_{\mathrm{w}}=84.3 \mathrm{kDa}$; PDI $=$ 1.94), 11-mercaptoundecanoic acid (MUA, 95\%), and membranes for dialysis $(7.0 \mathrm{kDa})$ were purchased from SigmaAldrich (USA). Sodium heparin (HEP) from porcine intestinal mucosa, containing $12.5 \%$ sulfur was purchased from Celsus Laboratories (USA). Glacial acetic acid and ethanol (>98 vol\%) were purchased from Acros Organics (USA). Sodium acetate was purchased from Fisher Scientific (USA). Phosphate buffered saline solution (PBS) without calcium and magnesium was purchased from Gibco (USA). A Millipore water purification unit was used to obtain $18.2 \mathrm{M} \Omega \mathrm{cm}$ water, used for making all aqueous solutions (Millipore, Billerica, MA).

\section{Purification of the amino-functionalized tannin (TN)}

The purification of the TN is carried out following a previously reported procedure ${ }^{3}$ with alterations. A TN solution $(50 \mathrm{~mL}$ at $10 \% \mathrm{wt} / \mathrm{vol}$ ) was prepared in ultrapure water, filtered and dialyzed for two days against ultrapure water, exchanging the water once per day. After dialysis, the polymer solution was frozen and lyophilized for $72 \mathrm{~h}$. 


\section{Construction of polyelectrolyte multilayers (PEMs)}

$\mathrm{TN}, \mathrm{HEP}$, and CS solutions at $1.0 \mathrm{mg} \mathrm{mL^{-1 }}$ were prepared in $0.2 \mathrm{M}$ acetate buffer solution $(\mathrm{pH}$ 5.0). A rinse solution was prepared from aqueous acetic acid at $\mathrm{pH}$ 4.0. All solutions were filtered with $0.22 \mu \mathrm{m}$ polyvinylidene fluoride (PVDF) syringe filters (Fisher Scientific). The LbL deposition was carried out on the oxidized glass surface (substrate disks of $8 \mathrm{~mm}$ diameter and $0.15 \mathrm{~mm}$ thickness). First, the glass surfaces were deposited inside sterile Nuclon $\Delta$ TCPS 24-well plates, Nunc ALS (Roskilde, Denmark) and then modified (oxidized using oxygen plasma) for promoting polycation deposition (TN). Before PEM deposition, the oxidized glass surfaces were rinsed by adding $1 \mathrm{~mL}$ rinse solution under shaking (100 rpm) for $6 \mathrm{~min}$. Then, the rinse solution was aspirated and $1 \mathrm{~mL} \mathrm{TN}$ (polycation) solution was added to the oxidized glass surface. After $6 \mathrm{~min}$, the polycation solution was aspirated and the surface was rinsed (under shaking for $6 \mathrm{~min}$ ) and aspirated. Then, CS or HEP (polyanion) solutions $(1.0 \mathrm{~mL})$ were deposited onto the oxidized glass surface containing one layer of TN (polycation). After assembling the second layer (polyanion), the process of deposition was repeated to achieve 10-layer (polyanionterminated) or 11-layer (TN-terminated) PEMs. Polyanionterminated ( $\mathrm{HEP}-\mathrm{TN}_{10}$ or $\left.\mathrm{CS}-\mathrm{TN}_{10}\right)$ and polycation-terminated $\left(\mathrm{HEP}-\mathrm{TN}_{11}\right)$ PEMs were constructed using 10 and 11 layers, respectively. Fig. 1 shows a schematic representation of the PEM preparation and chemical structures of the polyelectrolytes.

\section{Characterization}

In situ Fourier-transform surface plasmon resonance (FTSPR). Construction of PEMs on gold-coated glass chips with $47 \mathrm{~nm}$ thickness (NanoSPR LLC, Chicago, IL) has been previously studied in our laboratory. ${ }^{47}$ Gold-coated SF-10 glass chips were rinsed exhaustively in ethanol, then modified with a selfassembled monolayer of MUA by soaking the gold-coated glass chips in a $1 \mathrm{mM}$ MUA solution in ethanol for $24 \mathrm{~h}$. The intensity of polarized light reflected from the back side of the gold film on which the PEMs were assembled was measured as a function of wavenumber. Through the changes in the refractive index, associated with polyelectrolyte adsorption events, it is possible to monitor the LbL assembly of the PEM in situ. LBL assembly of PEM was conducted in the flow cell of an SPR-100 module coupled to a Nicolet 8700 FT-IR spectrometer (Thermo-Electron, Madison, WI). A Masterflex peristaltic pump was used to flow polycation, polyanion and rinse solutions through the flow cell at $1 \mathrm{~mL} \mathrm{~min}^{-1}$ flow rate. FT-SPR was performed using a white light/near-infrared source with a $\mathrm{CaF}_{2}$ beam splitter at the interferometer and an InGaS detector. Data were collected using the Omnic 7.3 software (Thermo Electron), at $8 \mathrm{~cm}^{-1}$ resolution over the range from $6000 \mathrm{~cm}^{-1}$ to $12000 \mathrm{~cm}^{-1}$. The center of gravity method provided in OMNIC was used to determine SPR peak position at each time point. PEMs were assembled by successively flowing solutions through the flow cell, for six minutes each, in the following sequence:
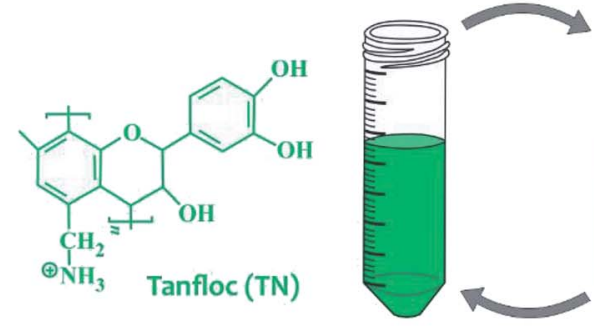

Polycation

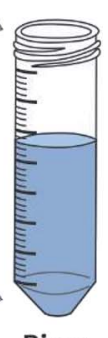

Rinse

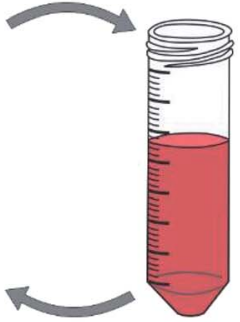

Polyanion

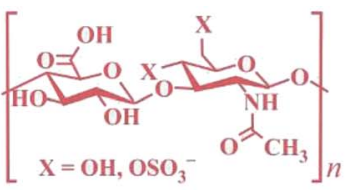

Chondroitin 4-, 6-Sulfate

or

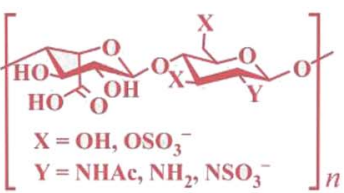

Heparin
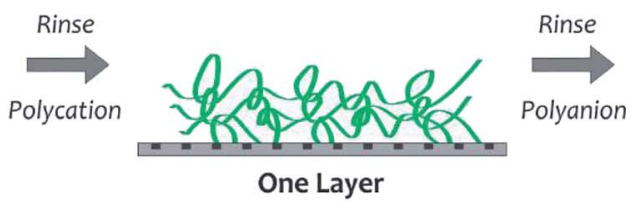

Substrate

One Layer

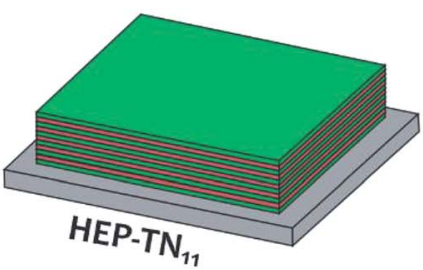

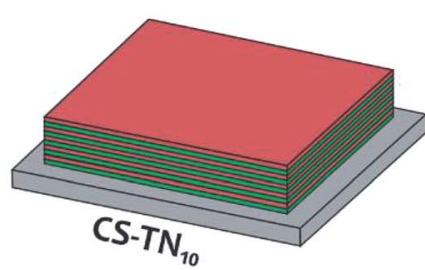

Fig. 1 Schematic representation for the PEM construction and chemical structures of the polyelectrolytes. 
polycation, rinse, and polyanion, rinse, until 11 layers had been deposited.

X-ray photoelectron spectroscopy (XPS). Surface chemistry of the PEMs was assessed via XPS using a Physical Electronics 5800 spectrometer (Chanhassen, MN). This system has a monochromatic $\mathrm{Al} \mathrm{K} \alpha \mathrm{X}$-ray source $(h \nu=1486.6 \mathrm{eV})$, hemispherical analyzer, and multichannel detector. The $\mathrm{C} 1 \mathrm{~s}$ peak at $284.8 \mathrm{eV}$ was used as reference for the samples. High-resolution spectra were obtained using a $23.5 \mathrm{eV}$ analyzer pass energy with $0.1 \mathrm{eV}$ steps and an X-ray spot of $800 \mu \mathrm{m}$. All spectra were obtained with a photoelectron take-off angle of $45^{\circ}$. A low-energy electron gun was used for charge neutralization. Spectral curve fitting using Guassian curves was done using Origin version 8.5 and Multipak (version 9.3.0.3, Ulvac-Phi, Inc.) software with a Shirley background correction.

Atomic force microscopy (AFM) and scanning electron microscopy (SEM). AFM was performed to evaluate surface topography and roughness of the PEMs, on a Bioscope Resolve AFM (Bruker) with ScanAsyst Air probes. The measurements were performed under ambient conditions, room temperature in air at $1 \mathrm{~Hz}$ line rate. AFM images were obtained using tapping mode from a scanning image probe processor version 4.2.2.0 software. For SEM, the samples were coated with palladiumgold alloy (Polaron SC 7620 Sputter Coater, Quorum Technologies, Newhaven, UK) at a thickness of $10 \mathrm{~nm}(10-15 \mathrm{~mA}$, under a vacuum of 130 mTorr). The SEM (JSM-6500F, field emission scanning electron microscope, JEOL, Japan) was operated at an accelerating voltage of $5 \mathrm{kV}$.

Water contact angle (WCA) measurements. Wettability of the PEMs was assessed through static contact angle measurements by the sessile drop method with a contact angle goniometer (Krüss DSA 10, Hamburg, Germany), equipped with video capture. The automatic dosing feature of the DSA 10 dispenses a water drop on the PEMs membrane surfaces, and the needle is manually withdrawn. Images were captured after $5 \mathrm{~s}$ of contact of the droplet with the sample using a camera leveled with the surface. Using the circle fitting profile in the DSA 10 imaging software, WCAs were estimated. Three separate measurements were made on each sample at different locations.

\section{Cytocompatibility assays}

Cell culture. Dr Kimberly Cox-York from the Department of Food Science and Human Nutrition at Colorado State University kindly donated adult human adipose-derived stem cells. They were cultured at $37{ }^{\circ} \mathrm{C}$ in a $5 \% \mathrm{CO}_{2}$ atmosphere in $175 \mathrm{~cm}^{2}$ surface area tissue-culture polystyrene flasks. Before cell seeding, samples were rinsed 3 times using PBS and then exposed to UV light for $30 \mathrm{~min}$. Cells were seeded onto PEM-modified glass surfaces (substrate disks, $8 \mathrm{~mm}$ diameter and $0.15 \mathrm{~mm}$ thickness) at 5000 cells per well in 48-well plates, and were cultured at $37^{\circ} \mathrm{C}$ in a $5 \% \mathrm{CO}_{2}$. Cells were cultured in Dulbecco's Modified Eagle Medium (DMEM) supplemented with 10\% fetal bovine serum and $1 \%$ penicillin/streptomycin. HEP-TN 10, CS-TN 10 and HEP-TN ${ }_{11}$ PEMs samples were used in triplicate $(n=3)$.

Cell viability assay. Cell viability was determined by a CellTiter-Blue ${ }^{\circledR}$ cell viability assay (Promega G808A, Madison, WI), following the manufacturer's instructions. After 1, 4 and 7 days $50 \mu \mathrm{L}$ of CellTiter-Blue dye were added to each experimental well, containing $500 \mu \mathrm{L}$ culture media. After $6 \mathrm{~h}$ incubation at $37{ }^{\circ} \mathrm{C}$ in $5 \% \mathrm{CO}_{2}$ atmosphere, the absorbance of the samples were read in a microplate reader (Molecular Devices Spectra Max M3, Sunnyvale, CA) at $570 \mathrm{~nm}$ and $600 \mathrm{~nm}$.

Adhesion and proliferation tests. The ADSC cell response on PEMs during 1, 4 and 7 days of cell culture was investigated using fluorescence microscopy. Cell adhesion and proliferation were characterized by fluorescence microscopy (Zeiss Axioskop 2 epifluorescence microscope). The cell cytoplasm was stained with 5-chloromethylfluorescein diacetate (CMFDA, Life Technologies), the cytoskeleton was stained using rhodamine phalloidin, and the cell nuclei were stained with 4',6-diamidino-2phenylindole (DAPI, Invitrogen). Prior to staining, the unadhered cells were aspirated, and substrates were gently rinsed with PBS (twice), and then transferred to a new 48-well plate before staining. Samples were then incubated at $37^{\circ} \mathrm{C}$ in $5 \%$ $\mathrm{CO}_{2}$ in a $10 \mu \mathrm{mol} \mathrm{L}{ }^{-1}$ solution of CMFDA in PBS for $45 \mathrm{~min}$. After incubation, the CMFDA solution was aspirated and substrates were incubated in a growth media $\left(37^{\circ} \mathrm{C}\right.$ at $\left.5 \% \mathrm{CO}_{2}\right)$ for $30 \mathrm{~min}$. The growth media solution was aspirated and the surfaces were again rinsed using PBS, before being transferred to a new 48-well plate. An aqueous $3.7 \% \mathrm{wt} / \mathrm{vol}$ formaldehyde solution (15 $\mathrm{min}$ at room temperature) was used to fix the cells. Fixative solution was then aspirated and substrates were rinsed with PBS (thrice), for 5 min per rinse, and transferred to a new 48-well plate. The cells were permeabilized in an aqueous $1.0 \%$ $\mathrm{vol} / \mathrm{vol}$ Triton-X solution (3 min and room temperature). After aspirating the Triton-X solution, the surfaces were rinsed with PBS and transferred to a new 48-well plate. Samples were then incubated (at $37{ }^{\circ} \mathrm{C}, 5 \% \mathrm{CO}_{2}$ ) with $5 \mu \mathrm{L} \mathrm{mL} \mathrm{m}^{-1}$ rhodaminephalloidin solution in deionized water for $25 \mathrm{~min}$. Next, a solution of $1 \mu \mathrm{L} \mathrm{mL}^{-1}$ of DAPI was added, and the samples were incubated for an additional $5 \mathrm{~min}$ at room temperature. The solution was aspirated, substrates were rinsed twice with PBS, and kept in a light-resistant container at $20{ }^{\circ} \mathrm{C}$ until imaging. Fluorescence images were analyzed and processed with Image software.

Cell morphology. Scanning electron microscopy (SEM) was used to access ADSC cell morphologies on the PEMs. After 7 days of cell culture, the samples containing the ADSC cells were fixed in $3 \% \mathrm{vol} / \mathrm{vol}$ glutaraldehyde, $0.1 \mathrm{M}$ sodium cacodylate and $0.1 \mathrm{M}$ sucrose solution for $45 \mathrm{~min}$. Samples were soaked in a buffer solution of $0.1 \mathrm{M}$ sodium cacodylate and $0.1 \mathrm{M}$ of sucrose for $10 \mathrm{~min}$. The fixed samples were processed in serial ethanol dehydrations (10 min each) followed by immersion in hexamethyldisilazane $(10 \mathrm{~min}$ ) before being stored until SEM analysis. Samples were sputter-coated with palladium-gold alloy at a thickness of $10 \mathrm{~nm}$ (10-15 mA, under a vacuum of 130 mTorr). The SEM was operated at an accelerating voltage of 15 $\mathrm{kV}$.

\section{Statistical analysis}

Values are expressed as mean \pm standard deviation. Comparisons between groups were performed using ANOVA and with 
Tukey's multiple comparison test, performed with Graph Prism 7.0, GraphPad Software Inc., La Jolla, CA, USA.

\section{Results and discussion}

\section{Layer-by-layer assembly and characterization of PEMs}

The layer-by-layer assembly of the TN-HEP was monitored by in situ Fourier-transform surface plasmon resonance (FT-SPR). Fig. 2 shows a representative results obtained during layer-bylayer by assembly of TN (polycation) and HEP (polyanion) PEM. We have previously shown that HEP and CS result in similar thicknesses for PEMs when paired with the weak, amine-containing polycation, chitosan. ${ }^{13}$ The first 6 min (blue arrow) represents rinse on the gold-coated glass chip modified with MUA. The red arrow indicates the beginning of the next 6 min interval when the polycation solution (TN) is introduced into the flow cell. The large drop in the FT-SPR peak position during this adsorption step is due to the adsorption of polycation to the surface and to the difference in refractive index between the rinse and polycation solutions as we reported previously. ${ }^{47}$ After the initial TN adsorption, the surface was rinsed for $6 \mathrm{~min}$; during this rinse the FT-SPR peak returns to a higher peak position. The net change in peak position before and after the TN adsorption step is due to the irreversible adsorption of positively charged polyelectrolyte. The green arrow indicates the beginning of the next 6 min interval when the polyanion solution (HEP) is introduced into the flow cell. This sequence was repeated until an 11-layer PEM was constructed.

The adsorption of each bilayer is characterized by a relatively large change in the SPR peak position following TN deposition $\left(>200 \mathrm{~cm}^{-1}\right)$, compared to the change associated with the HEP deposition $\left(>50 \mathrm{~cm}^{-1}\right)$. This could be due to the much higher molecular weight of the TN, compared to the HEP. The results observed here are consistent with the observations of Boddohi et al. who attributed relatively thin layers of HEP to an extended conformation adopted by HEP in solution, whereas weak

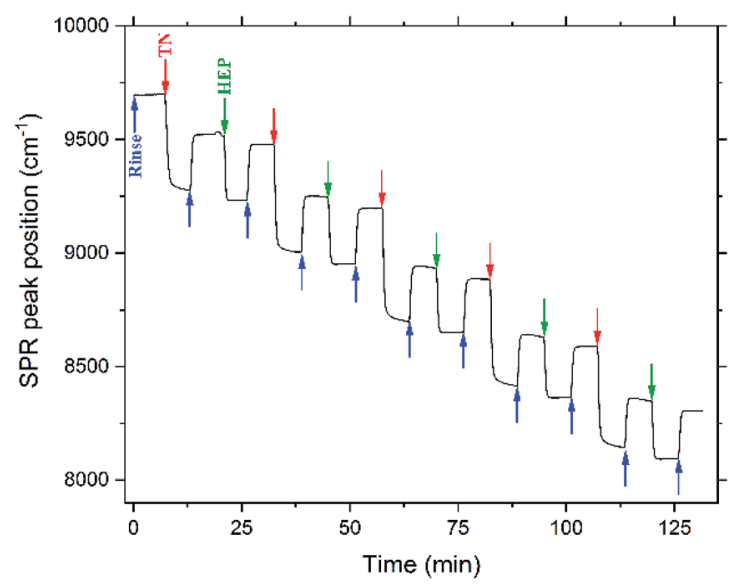

Fig. 2 PEM assembly monitored by in situ FT-SPR. Arrows represent the beginning of acidified water rinse (blue), TN deposition layers (red), and HEP deposition layers (green). polycations, like $\mathrm{TN}$, adopt a coil conformation in solution, and adsorb in thicker layers. ${ }^{\mathbf{1 3 , 4 7}}$

Previous work from our group has shown, using a combination of experiment and theory, that these polysaccharide-based PEMs are very thin, only a few nanometers. We used different polyanion-polycation pairs (chitosan (CHI)-hyaluronic acid (HA), CHI-CS, CHI-HEP and $N, N, N$-trimethyl chitosan (TMC)HA, TMC-CS, TMC-HEP) and thickness values varied in a range from $10 \mathrm{~nm}$ to $27 \mathrm{~nm}$ for 10 layers, depending on the interactions between strong and weak polyelectrolyte pairing. In this work, we showed that a strong polycation (TMC) results in thinner layers, whereas weak and a strong polyelectrolyte pairing (CHI-CS, CHI-HEP, and TMC-HA) result in thicker PEMs. The thickness of the adsorbed PEM also depends on $\mathrm{pH}$ conditions and ionic strength. Lower $\mathrm{pH}$ tends to increase PEM thickness values, probably associated with the increase of ammonium ionized groups in the weak polycation, as the $\mathrm{pH}$ is reduced. We also found that higher concentrations of sodium and acetate ions (in the buffer solution) lead to a significant screening of the electrostatic charges along polymer chains, resulting in thicker PEM films. ${ }^{13,15}$

The wettability of the TN-GAG PEM films was investigated through water contact angle (WCA) measurements. PEMs in this work are assembled under acid conditions, imparting ionized groups on the polyelectrolytes. The $\mathrm{p} K_{\mathrm{a}}$ of the amine groups $\left(-\mathrm{NH}_{2}\right)$ in $\mathrm{TN}$ is approximately 6.0 and the $\mathrm{p} K_{\mathrm{a}}$ of the carboxylate groups $\left(-\mathrm{COO}^{-}\right)$and sulfate groups $\left(-\mathrm{SO}_{3}{ }^{-}\right)$in the GAGs are 2-3 and 0.5-1.5, respectively. ${ }^{31,44}$ PEMs are very hydrophilic (WCAs of $28.6^{\circ} \pm 4.4^{\circ}, 26.3^{\circ} \pm 2.9^{\circ}$ and $38.3^{\circ} \pm 1.8^{\circ}$ for CS-TN $10, \mathrm{HEP}-\mathrm{TN}_{10}$ and $\mathrm{HEP}-\mathrm{TN}_{11}$, respectively). WCAs can also be correlated with the swelling capacity. Low WCAs are associated with high swelling ratios, since hydrophilic PEMs absorb more water, increasing thickness in solution. The WCA results here are in agreement with other reports of $\mathrm{LbL}$ assembly by using polysaccharide-based PEMs., ${ }^{5,26}$ Hydrophilic or high-surface energy substrates may improve cell adhesion and cell spreading. ${ }^{48-50}$

\section{X-ray photoelectron spectroscopy (XPS)}

The chemistry of PEM surfaces was characterized using survey and high-resolution XPS spectra. All XPS survey spectra show presence of carbon (C 1s at $287 \mathrm{eV}$ ), oxygen (O 1s at $534 \mathrm{eV}$ ) nitrogen ( $\mathrm{N}$ 1s at $401 \mathrm{eV}$ ), and sulfur (S $2 \mathrm{p}$ at $168.5 \mathrm{eV}$ ), confirming the assembling of GAGs and TN on oxidized glass surfaces (Fig. 3A). No observable detection of a silicon peak demonstrates complete coating of the substrate using 10 layers of the polyelectrolytes. The TN-terminated PEM surface shows a chlorine peak ( $\mathrm{Cl} 1 \mathrm{~s}$ at $200 \mathrm{eV}$ ), probably associated with counter-ions of the TN. Sodium peaks also appear in some XPS spectra due to presence of sodium in acetic acid/sodium acetate buffer solution used to create the polyelectrolyte solutions. Sodium ions interact with carboxylate and sulfated moieties on GAGs by electrostatic interactions. Table $\mathrm{S} 1 \dagger$ indicates the atomic composition of the samples (ESI $\dagger$ ).

Chemical characterization of TN has been described previously. ${ }^{3}$ High-resolution XPS spectra for N 1s and S 2p envelopes 
A

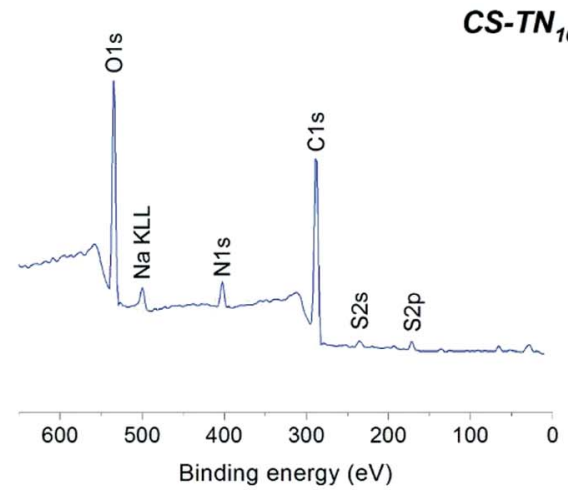

$\stackrel{\infty}{\circ}$
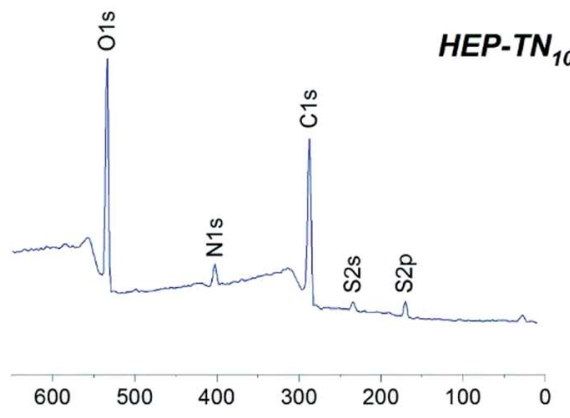

Binding energy (eV)

B
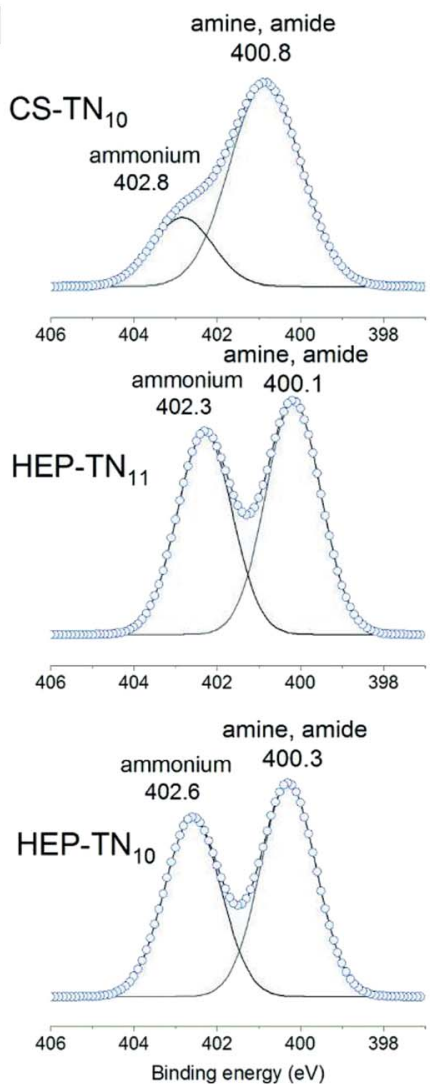
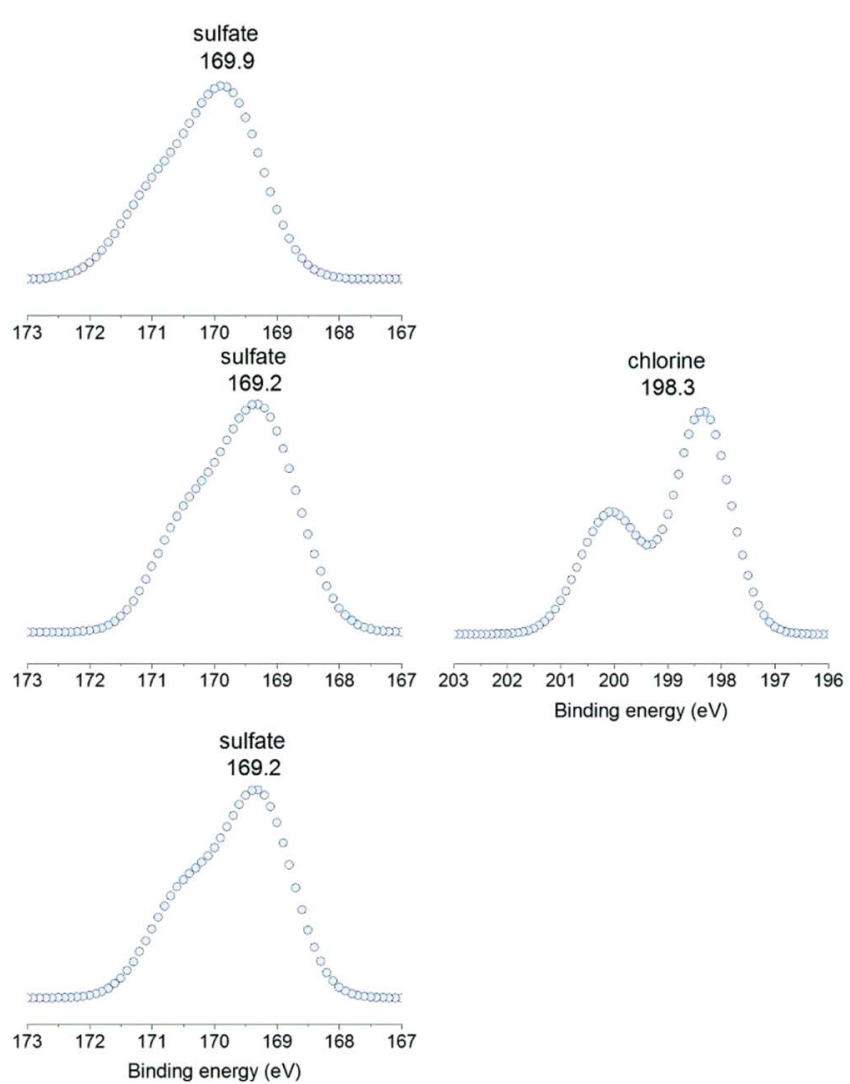

Fig. 3 Survey (A) and high-resolution $\mathrm{X}$-ray spectra (B) of the $\mathrm{N} 1 \mathrm{~s}, \mathrm{~S} 2 \mathrm{p}$ and $\mathrm{Cl} 2 \mathrm{~s}$ envelopes determined on PEM surfaces.

(Fig. 3B) identified characteristic chemical groups found on TN, HEP, and CS structures. PEM surfaces exhibit amine (399.6 eV), amide $(400.7 \mathrm{eV})$ overlapping with the position of the amine, and ammonium peaks $(402 \mathrm{eV})$. The protonated nitrogen at higher binding energy indicates ionic interactions between the nitrogen groups of the polyelectrolytes and small counterions in 
the PEM structures. Since sulfur atoms do not occur in TN structure, the $\mathrm{S} 2 \mathrm{p}$ envelope is related to the sulfate groups in the GAGs (HEP and CS), which confirm addition of HEP and CS in the PEMs. High-resolution spectra for the $\mathrm{C}$ 1s envelope (Fig. S.1, ESI $\dagger$ ) show aliphatic carbon $(\sim 284.8 \mathrm{eV})$; alcohol, amine and ether (at $\sim 287 \mathrm{eV}$ ), amide $(\sim 289 \mathrm{eV})$, and the carboxylic acid $(\sim 289 \mathrm{eV})$.

\section{Atomic force microscopy (AFM)}

Surface topography plays an essential role in determining protein adsorption and cell adhesion to biomaterials applied as medical devices. By modulating protein and cell interactions through surface topography, important biological responses such as bacterial cell adhesion, stem cell adhesion, and platelet adhesion can all be affected. ${ }^{5,9,10}$ The topological features of the PEMs were characterized in air by AFM. The images of multilayer films prepared on the glass substrate and assembled at $\mathrm{pH}$ 5.0 are shown in Fig. 4. The surfaces were very smooth with roughness average of $5.7 \pm 1.5 \mathrm{~nm}$ owing to the strong association between TN and GAGs. The CS-TN 10 PEM had slightly higher roughness $(7.1 \pm 0.2 \mathrm{~nm})$. The mean squared roughness $\left(R_{\mathrm{RMS}}\right)$ and average height value $\left(H_{\mathrm{AV}}\right)$ depend on the polyelectrolyte interactions and polymer conformation..$^{29}$ Previous reports have shown strong associations between weak polycations (chitosan) with strong polyanions (HEP and CS). ${ }^{13}$ The coiled conformation, usually adopted by weakly charged polyelectrolytes lead to rougher surfaces, which could explain why the CS-terminated $\left(\mathrm{CS}-\mathrm{TN}_{10}\right)$ PEM surface has higher roughness compared to the HEP-based PEMs, since HEP has more sulfated groups. ${ }^{51}$ The higher molecular weight of CS could also contribute to the increased roughness. AFM images corroborate complete surface coverage, and confirm the homogeneous polyelectrolyte deposition and assembly of the TN-GAG multilayered films.

\section{Cell viability}

Cytocompatibility of the PEMs was evaluated in vitro by using AlamarBlue reagent through cell viability measurements (Fig. 5). Viable cells are able to reduce the active agent of
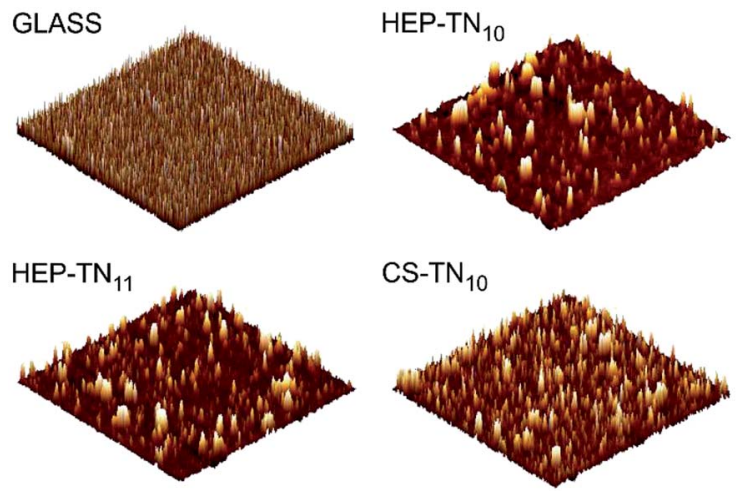

Fig. 4 Representative $10 \mu \mathrm{m} \times 10 \mu \mathrm{m}$ AFM topographic images of the glass (non-modified), CS-TN $10, \mathrm{HEP}-\mathrm{TN}_{10}$ and HEP-TN 11 surfaces.

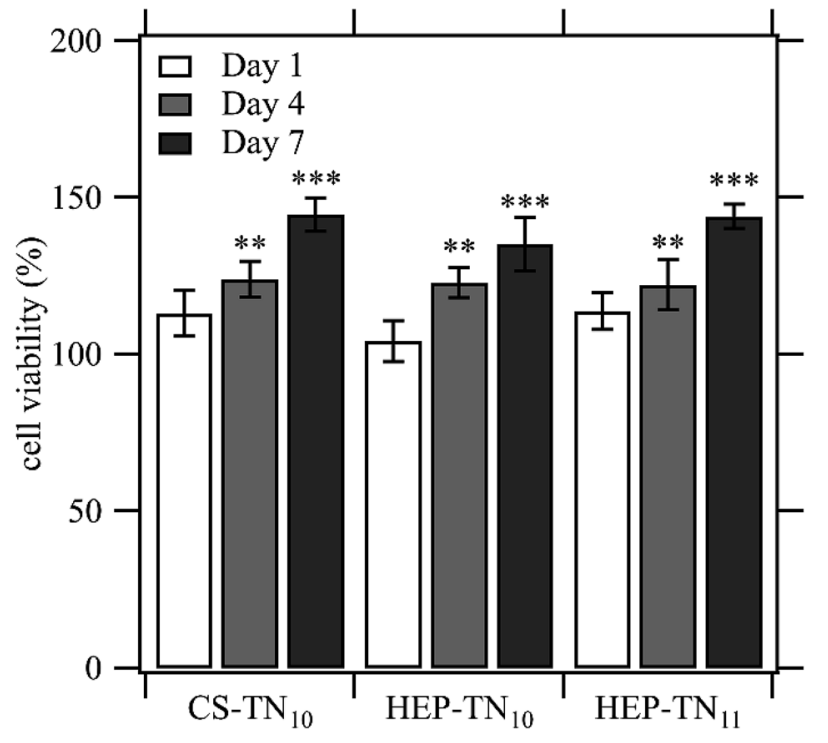

Fig. 5 Effect of the contact with CS-TN $10, \mathrm{HEP}-\mathrm{TN}_{10}$ and $\mathrm{HEP}-\mathrm{TN}_{11}$ on ADSC activity normalized to the control (cell culture with standard supplemented culture medium on TCPS). Cell viability at day 4 and day 7 were statistically different from the control on days 4 and $7(n=5$; ** indicates $p<0.01$ compared to the control; *** indicates $p<0.0001$ compared to the control).

AlamarBlue (resazurin dye), resulting in a quantifiable color change from blue to pink. ${ }^{52}$ The number of living cells is proportional to the absorption of the dye. Cell viability on the PEMs was evaluated after 1, 4 and 7 days of cell culture. The results show an increase in cell activity from day 1 to day 7 for all samples, indicating cell proliferation. CS- $\mathrm{TN}_{10}$ presented 144.5 $\pm 5.4 \%$ cell viability, followed by HEP-TN ${ }_{11} 144 \pm 3.8 \%$ and HEP-TN ${ }_{10} 135 \pm 8.5 \%$ at day 7 with respect to the control (cell culture on TCPS with standard supplemented culture medium). Significant statistical differences were observed at day 7 between groups and control. Cell viabilities occurring at $80 \%$ confirm suitable cytocompatibility, adhesion and proliferation for ADSCs. $^{40,52}$ ISO 10993-549 states that reduction of cell viability by more than $30 \%$ is considered cytotoxic. Therefore all PEMs in this work are non-cytotoxic toward ADSCs. Non-toxicity is an essential feature of microenvironments for cell adhesion and growth. ${ }^{50,53}$

Peniche and co-workers obtained films of CHI-HA and CHICS with very homogeneous, compact and smooth surfaces, which were prepared by a casting method. The viability of fibroblasts on these samples was evaluated using standard biological MTT and Alamar Blue tests. ${ }^{40}$ They observed that there is specific activation of the proliferative process in the presence of GAGs. Wilson and co-workers used poly(styrene sulfonate) and poly(acrylic acid) PEMs to minimize gold nanorods (AuNRs) cytotoxicity on fibroblast cells and improve interactions with proteins found in the ECM. Both polyanionterminated and polycation-terminated PEM-coated AuNRs enhanced cell viabilities. It was concluded that the PEMs influence cell behavior via multiple mechanisms, depending on 
the terminal layer, resulting from different interactions with proteins found in the ECM. ${ }^{54}$

There are few reports of biomaterials based on $\mathrm{TN}$ for biomedical applications. Recent work shows that $\operatorname{poly}(\varepsilon-$ caprolactone)/TN nanofibers produced via electrospinning and containing TN content (up to $22 \mathrm{wt} \%$ ), increased the wettability of the nanofiber surface, making it more favorable for cell adhesion and proliferation. ${ }^{3}$ Also, the hydrophilic groups in the TN structure, such as $-\mathrm{NH}_{2} /-\mathrm{NH}_{3}{ }^{+}$and $-\mathrm{OH}$ play an essential role in cell anchorage, conferring bioadhesivity to the nanofibers and an improved microenvironment to promote cell survival. ${ }^{3}$

\section{Adhesion and proliferation assays}

ADSC adhesion and proliferation tests were assessed after 1, 4 and 7 days of cell culture through fluorescence microscopy, by staining cells with CMFDA, rhodamine-phalloidin, and DAPI for imaging cytoplasm, cytoskeleton and nuclei, respectively. Samples were seeded at a very low cell density (5000 per well). Ten non-overlapping images were taken $20 \times$ magnification for each surface type in triplicate $(n=3)$ in each time point. The microscopy images indicate cell growth during 1 to 7 days of cell culture (Fig. 6). The results showed adhesion of the ADSCs and surface coverage for all samples. Cells are spreading out, confirming AlamarBlue results of cell viability and proliferation.

Scanning electron microscopy (SEM) was used to evaluate the cell morphology. Fig. 7 shows overview and higher resolution images after 7 days of cell culture. It underlines the former results of fluorescence microscopy and Alamar Blue test. Cells with different morphologies are interacting with the films and show clear fibroblastic morphologies on all PEM surfaces.

DAPI binds DNA adenine-thymine regions and cell proliferation can be estimated by calculating the number of nuclei adhered to the PEM surfaces (Fig. 8). Cell count per area corroborate the findings obtained by analyzing the fluorescence microscopy images. All experimental surfaces have more cells adhered than the control (unmodified glass) at day 4 and day 7 . Cells on the CS-terminated $\left(\mathrm{CS}-\mathrm{TN}_{10}\right)$ PEM were less spread compared to the other PEM surfaces, not covering the entire surface, leaving some unpopulated spaces. This could be due to PEM erosion caused during the preparation of the samples for capture of the fluorescence images. The high concentration of cells suggest formation of aggregates compensating with cellcell adhesion. ${ }^{55}$ HEP PEMs tend to promote more cell
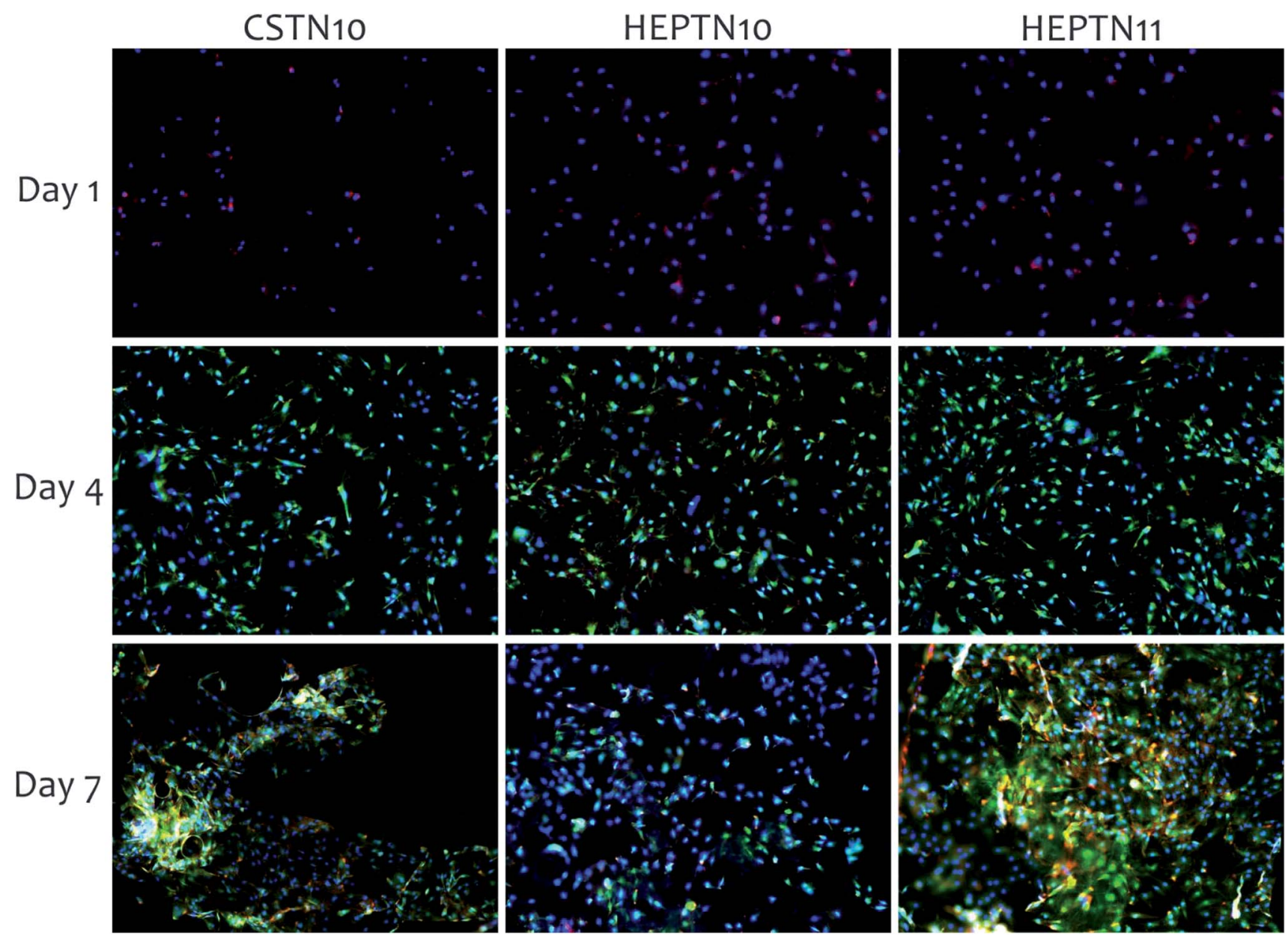

Fig. 6 Fluorescence microscopy images of ADSC cells to visualize cytoplasm (green), cytoskeleton (red), and nucleus (blue) on CS-TN 10 , HEP$\mathrm{TN}_{10}$ and HEP-TN 11 PEMs. The figure shows day 1, 4 and 7 of cell culture. 


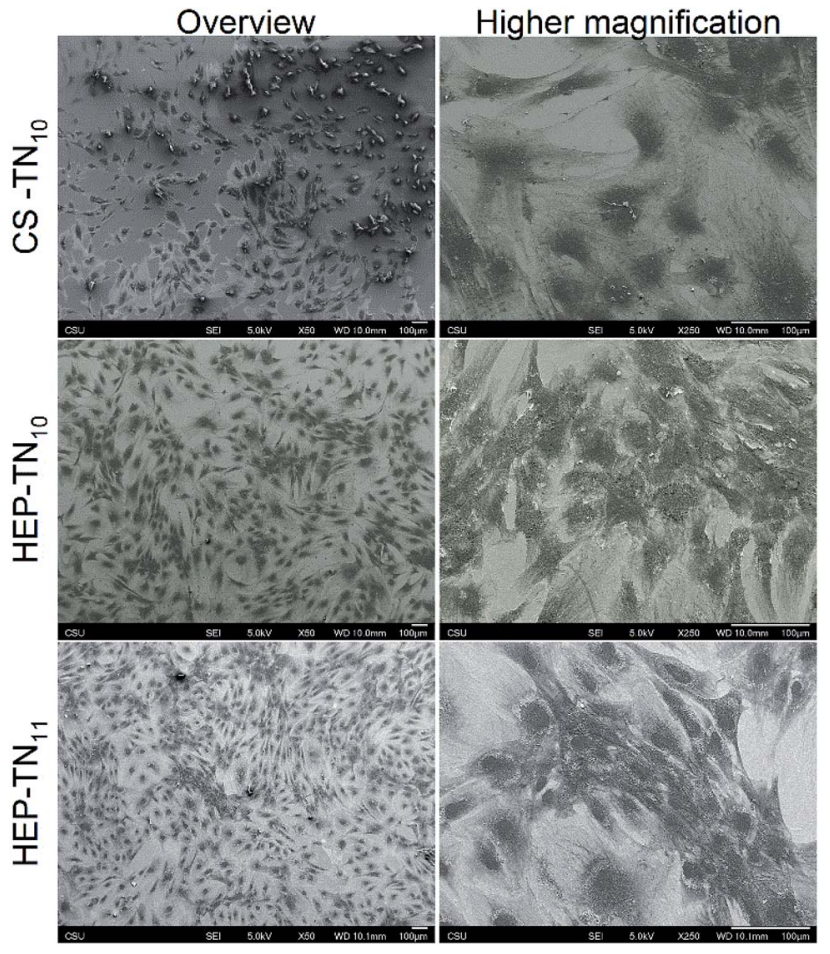

Fig. 7 SEM overview and higher magnification micrographs of ADSCS seeded on CS-TN 10, HEP-TN 10 and HEP-TN 11 PEMs, after 7 days of cell culture.

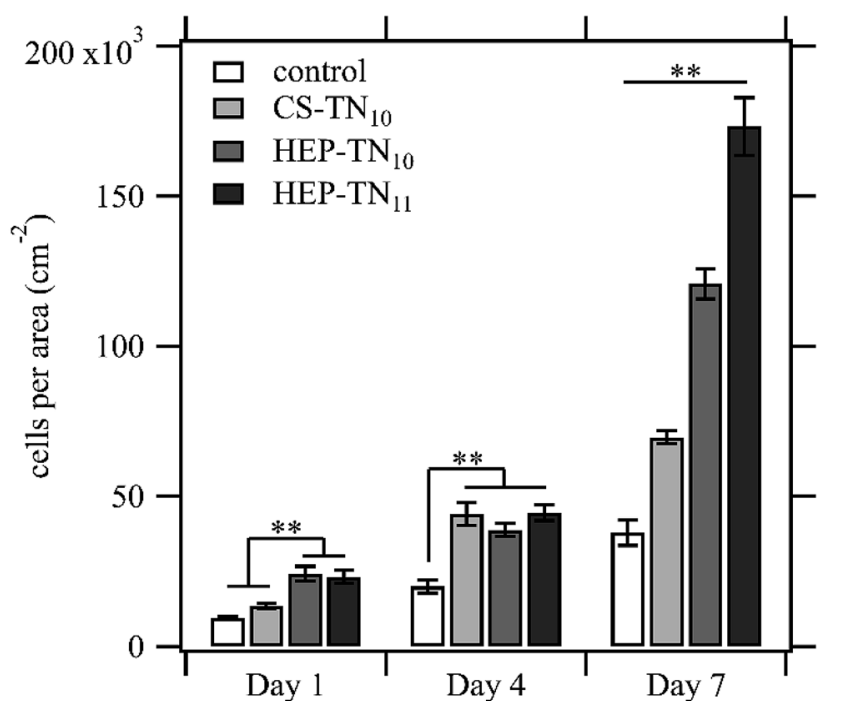

Fig. 8 Quantification of ADSC cells per area on control (unmodified glass), CS-TN $10, \mathrm{HEP}-\mathrm{TN}_{10}$ and HEP-TN 11 , after 1,4 and 7 days of cell culture. Significant differences between different surface types on the same day are indicted $(n=5 ; * *$ indicates $p<0.01)$.

proliferation than CS PEMs and TN-terminated (HEP-TN $\left.{ }_{11}\right)$ PEMs had significantly higher cell density per area after 7 days of culture $(p<0.01)$, indicating that the terminal TN layer positively influences the cell attachment and proliferation. Also, there is a favorable tendency of ADSCs to adhere and proliferate on positively charged substrates. ${ }^{24}$ Negatively charged osteoblasts were also reported to attach and proliferate more favorably on positively charged surfaces. ${ }^{56}$ The predominance of negatively charged species in the mammalian cell membrane exert electrostatic attraction when the final layer is positively charged, which may explain why the HEP-TN $\mathrm{TN}_{11}$ exhibits a significant and greater number of cells attached to the surface.$^{\mathbf{8} 57}$ Protonated amino groups on TN may be responsible for a bioadhesive characteristic, enhancing the cell attachment. Similar results were identified by Denuziere and co-workers, using chitosan-chondroitin sulfate and chitosan-hyaluronate polyelectrolyte complexes. ${ }^{41}$

A study of Boura and co-workers demonstrates the important role of PEM surface charge to cell response using human endothelial cells on LbL films of poly(sodium-4styrenesulfonate)/poly(allylamine hydrochloride). They also found that cells adhere and spread more favorably on polycation-terminated films. ${ }^{58}$ However, there are many aspects involved in cell-biomaterial interactions, and studies using other cells and different adhesion mechanisms suggests other outcomes. ${ }^{59,60}$ This reinforces the versatility of the LbL method of preparing thin films, which can be used to adjust key chemical and physical properties of the surface to influence cell adhesion.

Another important factor to long-term cell response is the water content. A higher amount of water could lead to weaker cell growth. The surface hydration makes the displacement of water molecules to enable cell and protein adhesion thermodynamically unfavourable. ${ }^{61}$ Aggarwal and co-workers using chitosan, HEP, cellulose sulfate, and myoblast cells, observed that CHI-HEP PEMs led to poor cell growth due to the higher amount of coupled water in the PEM structure. ${ }^{39}$ A similar phenomenon may be at work here. Since all PEMs studied here are hydrophilic, the increased hydrophilicity of the GAGterminated PEMs (with lowest WCAs) may cause the reduced cell adhesion and proliferation compared to TN-terminated PEMs.

Almodóvar and co-workers used chitosan and heparin to build polysaccharide-based polyelectrolyte multilayers to bind and stabilize growth factors (FGF-2) for delivery to bone-marrow derived mesenchymal stem cell (MSCs), thereby promoting cell proliferation and differentiation. ${ }^{38}$ The ability of heparin to bind and potentiate growth factors results in greater cell densities. Hence GAG-containing PEMs may be valuable for tissue engineering and surface coatings to enhance and guide cell responses to biomaterials.

\section{Conclusion}

This is the first report of polyelectrolyte multilayers formed from tanfloc and glycosaminoglycans through the LbL assembly method. The interactions of ADSCs with these new biomaterial surfaces demonstrate that these surface coatings exhibit good cytocompatibility and that they promote cell attachment and proliferation. These characteristics are very desirable for biocompatible coatings for applications such as tissue engineering scaffolds and orthopedic implants. The uses of tanfloc, 
instead of chitosan and its derivatives, is emerging as an alternative to overcome chitosan limitations (such as poor solubility in aqueous solutions) and to take advantage of the reduced cost of obtaining natural condensed tannins. These coatings could be used to enhance cell responses of cell resistant biomaterials, using a biologically sourced tanninderivative, which is in the nascent stages of development for medical devices and tissue engineering.

\section{Conflicts of interest}

There are no conflicts to declare.

\section{Acknowledgements}

The authors acknowledge Fulbright and CAPES for financial support through a PhD Scholarship for the first author. The authors also thank Dr Ellen Brennan-Pierce, Terrance Bishop for supporting the AFM instrument and Dr Patrick McCurdy assisting with XPS analysis.

\section{References}

1 J. G. Martins, S. E. A. Camargo, T. T. Bishop, K. C. Popat, M. J. Kipper and A. F. Martins, Carbohydr. Polym., 2018, 197, 47-56.

2 J. M. Silva, R. L. Reis and J. F. Mano, Small, 2016, 12, 43084342 .

3 A. F. Martins, S. P. Facchi, P. C. F. da Câmara, S. E. A. Camargo, C. H. R. Camargo, K. C. Popat and M. J. Kipper, J. Colloid Interface Sci., 2018, 525, 21-30.

4 R. Simon-Walker, R. Romero, J. M. Staver, Y. Zang, M. M. Reynolds, K. C. Popat and M. J. Kipper, ACS Biomater. Sci. Eng., 2017, 3, 68-77.

5 Y. Wang, Q. Hong, Y. Chen, X. Lian and Y. Xiong, Colloids Surf., B, 2012, 100, 77-83.

6 O. Urbanek, P. Sajkiewicz and F. Pierini, Polymer, 2017, 124, 168-175.

7 M. Hedayati, M. J. Neufeld, M. M. Reynolds and M. J. Kipper, Mater. Sci. Eng., R, 2019, 138, 118-152.

8 S. Guo, X. Zhu and X. J. Loh, Mater. Sci. Eng., C, 2017, 70, 1163-1175.

9 M. Padial-Molina, P. Galindo-Moreno, J. E. FernándezBarbero, F. O'Valle, A. B. Jódar-Reyes, J. L. Ortega-Vinuesa and P. J. Ramón-Torregrosa, Acta Biomater., 2011, 7, 771-778.

10 A. Ranella, M. Barberoglou, S. Bakogianni, C. Fotakis and E. Stratakis, Acta Biomater., 2010, 6, 2711-2720.

11 M. Votteler, P. J. Kluger, H. Walles and K. Schenke-Layland, Macromol. Biosci., 2010, 10, 1302-1315.

12 M. Keeney, X. Y. Jiang, M. Yamane, M. Lee, S. Goodman and F. Yang, J. Mater. Chem. B, 2015, 3, 8757-8770.

13 J. Almodóvar, L. W. Place, J. Gogolski, K. Erickson and M. J. Kipper, Biomacromolecules, 2011, 12, 2755-2765.

14 R. Aston, M. Wimalaratne, A. Brock, G. Lawrie and L. Grøndahl, Biomacromolecules, 2015, 16, 1807-1817.

15 M. Hedayati and M. J. Kipper, Carbohydr. Polym., 2018, 190, 346-355.
16 F. Z. Volpato, J. Almodovar, K. Erickson, K. C. Popat, C. Migliaresi and M. J. Kipper, Acta Biomater., 2012, 8, 1551-1559.

17 J. Almodóvar and M. J. Kipper, Macromol. Biosci., 2011, 11, 72-76.

18 S. Boddohi, J. Almodóvar, H. Zhang, P. A. Johnson and M. J. Kipper, Colloids Surf., B, 2010, 77, 60-68.

19 J. Almodovar, J. Mower, A. Banerjee, A. K. Sarkar, N. P. Ehrhart and M. J. Kipper, Biotechnol. Bioeng., 2013, 110, 609-618.

20 R. Romero, L. Chubb, J. K. Travers, T. R. Gonzales, N. P. Ehrhart and M. J. Kipper, Carbohydr. Polym., 2015, 122, 144-151.

21 J. Huang, X. Qiu, B. Yan, L. Xie, J. Yang, H. Xu, Y. Deng, L. Chen, X. Wang and H. Zeng, J. Mater. Chem. B, 2018, 6, 3742-3750.

22 J. J. Richardson, M. Bjornmalm and F. Caruso, Science, 2015, 348, aaa2491.

23 J. J. Richardson, J. Cui, M. Björnmalm, J. A. Braunger, H. Ejima and F. Caruso, Chem. Rev., 2016, 116, 14828-14867.

24 A. Nolte, S. Hossfeld, B. Schroeppel, A. Mueller, D. Stoll, T. Walker, H. P. Wendel and R. Krastev, J. Biomater. Appl., 2013, 28, 84-99.

25 S. del Hoyo-Gallego, L. Pérez-Álvarez, F. Gómez-Galván, E. Lizundia, I. Kuritka, V. Sedlarik, J. M. Laza and J. L. Vila-Vilela, Carbohydr. Polym., 2016, 143, 35-43.

26 J. H. Fu, J. Ji, W. Y. Yuan and J. C. Shen, Biomaterials, 2005, 26, 6684-6692.

27 B. Wang, K. Ren, H. Chang, J. Wang and J. Ji, ACS Appl. Mater. Interfaces, 2013, 5, 4136-4143.

28 R. J. Smith, M. G. Moule, P. Sule, T. Smith, J. D. Cirillo and J. C. Grunlan, ACS Biomater. Sci. Eng., 2017, 3, 1845-1852.

29 J. M. Silva, J. R. García, R. L. Reis, A. J. García and J. F. Mano, Acta Biomater., 2017, 51, 279-293.

30 M. Hedayati, M. M. Reynolds, D. Krapf and M. J. Kipper, ACS Appl. Mater. Interfaces, 2018, 10, 31892-31902.

31 T. Crouzier, T. Boudou and C. Picart, Curr. Opin. Colloid Interface Sci., 2010, 15, 417-426.

32 Y. Wei, H.-C. Hung, F. Sun, T. Bai, P. Zhang, A. K. Nowinski and S. Jiang, Acta Biomater., 2016, 40, 16-22.

33 S. Boddohi and M. J. Kipper, Adv. Mater., 2010, 22, 29983016.

34 D. H. Choi, S. N. Kang, S. M. Kim, S. Gobaa, B. J. Park, I. H. Kim, Y. K. Joung and D. K. Han, Colloids Surf., B, 2016, 141, 602-610.

35 L. W. Place, M. Sekyi and M. J. Kipper, Biomacromolecules, 2014, 15, 680-689.

36 L. W. Place, S. M. Kelly and M. J. Kipper, Biomacromolecules, 2014, 15, 3772-3780.

37 C. Lin, R. Romero, L. V. Sorokina, K. R. Ballinger, L. W. Place, M. J. Kipper and S. R. Khetani, J. Biomed. Mater. Res., Part A, 2018, 106, 971-984.

38 J. Almodóvar, S. Bacon, J. Gogolski, J. D. Kisiday and M. J. Kipper, Biomacromolecules, 2010, 11, 2629-2639.

39 N. Aggarwal, N. Altgärde, S. Svedhem, K. Zhang, S. Fischer and T. Groth, Colloids Surf., B, 2014, 116, 93-103. 
40 C. Peniche, M. Fernández, G. Rodríguez, J. Parra, J. Jimenez, A. L. Bravo, D. Gómez and J. San Román, J. Mater. Sci.: Mater. Med., 2007, 18, 1719-1726.

41 A. Denuziere, D. Ferrier, O. Damour and A. Domard, Biomaterials, 1998, 19, 1275-1285.

42 R. Romero, J. K. Travers, E. Asbury, A. Pennybaker, L. Chubb, R. Rose, N. P. Ehrhart and M. J. Kipper, J. Biomed. Mater. Res., Part A, 2017, 105, 900-911.

43 J. Lišková, T. E. L. Douglas, J. Beranová, A. Skwarczyńska, M. Božič, S. K. Samal, Z. Modrzejewska, S. Gorgieva, V. Kokol and L. Bačáková, Carbohydr. Polym., 2015, 129, 135-142.

44 N. Graham, F. Gang, G. Fowler and M. Watts, Colloids Surf., A, 2008, 327, 9-16.

45 Y. T. Hameed, A. Idris, S. A. Hussain and N. Abdullah, J. Environ. Manage., 2016, 184, 494-503.

46 D. P. Facchi, A. C. Lima, J. H. de Oliveira, D. Lazarin-Bidóia, C. V. Nakamura, E. A. Canesin, E. G. Bonafé, J. P. Monteiro, J. V. Visentainer, E. C. Muniz and A. F. Martins, Int. J. Biol. Macromol., 2017, 103, 129-138.

47 S. Boddohi, C. E. Killingsworth and M. J. Kipper, Biomacromolecules, 2008, 9, 2021-2028.

48 R. A. Gittens, R. Olivares-Navarrete, Z. Schwartz and B. D. Boyan, Acta Biomater., 2014, 10, 3363-3371.

49 S. P. Zhong, W. E. Teo, X. Zhu, R. Beuerman, S. Ramakrishna and L. Y. L. Yung, Mater. Sci. Eng., C, 2007, 27, 262-266.
50 P. Pulyala, A. Singh, M. F. Dias-Netipanyj, S. C. Cogo, L. S. Santos, P. Soares, V. Gopal, V. Suganthan, G. Manivasagam and K. C. Popat, Mater. Sci. Eng., C, 2017, 75, 1305-1316.

51 M. B. M. Spera, T. B. Taketa and M. M. Beppu, Biointerphases, 2017, 12, 04E401.

52 T. Greene and C.-C. Lin, ACS Biomater. Sci. Eng., 2015, 1, 1314-1323.

53 Y.-S. Li, Y. Han, J.-T. Qin, Z.-Y. Song, H.-H. Cai, J.-F. Du, S.-F. Sun and Y. Liu, J. Appl. Polym. Sci., 2016, 133, 44150.

54 C. G. Wilson, P. N. Sisco, F. A. Gadala-Maria, C. J. Murphy and E. C. Goldsmith, Biomaterials, 2009, 30, 5639-5648.

55 L. Bacakova, L. Grausova, J. Vacik, A. Fraczek, S. Blazewicz, A. Kromka, M. Vanecek and V. Svorcik, Diamond Relat. Mater., 2007, 16, 2133-2140.

56 Y. Zhang, W.-L. Gao, Z.-Y. Liu, Y. Jiang, K. Duan and B. Feng, Chin. Chem. Lett., 2016, 27, 1091-1096.

57 J. Vance and R. Steenbergen, Prog. Lipid Res., 2005, 44, 207234.

58 C. Boura, P. Menu, E. Payan, C. Picart, J. C. Voegel, S. Muller and J. F. Stoltz, Biomaterials, 2003, 24, 3521-3530.

59 C. Brunot, B. Grosgogeat, C. Picart, C. Lagneau, N. JaffrezicRenault and L. Ponsonnet, Dent. Mater., 2008, 24, 10251035.

60 S. Kidambi, I. Lee and C. Chan, J. Am. Chem. Soc., 2004, 126, 16286-16287.

61 J. B. Schlenoff, Langmuir, 2014, 30, 9625-9636. 